\title{
Does Computed Tomography of Kidney, Ureter and Bladder (CTKUB) Affect The Management of Patients with Suspected Renal Colic in Emergency Department?
}

Mehmood Chaudhry ( $\square$ mchaudhry@doctors.org.uk)

SKMC https://orcid.org/0000-0002-2766-3301

Rasha Buhumaid

SKMC: Sheikh Khalifa Medical City

Khulood A Al Haj Banimatar

SKMC

Ruqya Al Zaabi

SKMC

Ismail Al Ramahi

SKMC

Rayhan Chaudhry

Cardiff University

Original Research

Keywords: CTKUB, ED, patients

Posted Date: January 5th, 2021

DOI: https://doi.org/10.21203/rs.3.rs-133992/v1

License: (c) (i) This work is licensed under a Creative Commons Attribution 4.0 International License.

Read Full License 


\section{Abstract}

Introduction CTKUB use in patients presenting to Emergency Department (ED) with suspected renal colic has increased by 10 -fold over last few years. However, its impact on the urological intervention is not fully known.

Methods We performed a retrospective study of adult patients, presenting consecutively to ED with suspected renal colic who underwent CTKUB from January to June 2014. We calculated descriptive summary statistics for analysis to determine the association of predefined radiological and patients' characteristics on the urological intervention.

Results Of the 282 patients enrolled in the study, $38 \%$ had stones. Commonest locations of stone were ureter (15\%), uretero-vesical junction (12.4\%). Of the study participants, $2 \%$ of the patients underwent urological intervention. Chi Square analysis did not find any significant associations between radiological or patients related characteristics and urological intervention

Conclusion There was no significant correlation between CTKUB findings or patients' characteristics and urological intervention in this study cohort.

\section{Introduction}

Renal colic is a relatively common condition presenting to Emergency Department (ED) with greater than one million ED visits per year in the USA ${ }^{1,2}$. The classic presentation of urolithiasis is colicky flank pain that radiates to the groin. Gross or microscopic haematuria is often seen but its absence does not rule out the diagnosis. The majority of patients are managed conservatively in ED and up to $80 \%$ of calculi are passed spontaneously ${ }^{3}$. Over the last decade, non-contrast Computed Tomography of Kidney, Ureter and Bladder (CTKUB) has become the main imaging test for suspected renal colic due to its ready availability and high sensitivity and specificity in detecting the size and location of the stones. Despite a 10-fold increase in CTKUB usage in recent years, the proportion of patients diagnosed with urolithiasis and patients receiving urological intervention has not changed ${ }^{4}$. Many ED patients undergo CT for suspected renal colic without needing any urological intervention at the expense of over radiation and over diagnoses ${ }^{5}$. Although certain characteristics are cited as indications for possible urological intervention such as stone size of greater than $10 \mathrm{~mm}$, persistent pain, infection and renal impairment ${ }^{6}$; there is a paucity of evidence in proposing rational utilisation of CTKUB for suspected renal colic patients. The primary aim of our study was to evaluate the association of radiological findings and need for admission and urological intervention. Secondary aims were to analyse the association between patients' and radiological characteristics and a need for urological intervention in a cohort of ED patients with suspected renal colic.

\section{Material And Methods}


We conducted a retrospective descriptive study for consecutive patients presenting to our ED from January 2014 to June 2014. Sheikh Khalifa Medical City Hospital is a 650-bed tertiary hospital in the heart of Abu Dhabi City in the UAE. Our ED sees over 165,000 per year. This study was approved by the local institutional review board.

All patients aged 18 years or over who presented to ED during the study period with suspected renal colic and underwent CTKUB were included in the study. The decision to request CTKUB was left at discretion of attending physician for the patients presenting with classical history of renal colic. There were no specific exclusion criteria. All CT scans were performed without intravenous contrast and all CTs were reported by the consultant/specialist radiologist.

Study variables were abstracted from the electronic medical record (Cerner) including pathology and radiology reports. Statistical analysis was performed using SPSS. We calculated descriptive summary statistics for analysis. Patients characteristics included age, sex, nationality, presence of fever $(\mathrm{T}>38 \mathrm{C})$, onset of symptoms (within 6 hours or $>6$ hours), dysuria, haematuria (microscopic), vomiting, renal impairment (serum creatinine $>100 \mathrm{mmols} / \mathrm{L}$ ), presence of urinary nitrates. CTKUB characteristics included presence of significant hydronephnosis, and calculi including size and site. The size of stone was recorded as $<5 \mathrm{~mm},>5 \mathrm{~mm},>10 \mathrm{~mm}$ and locations as renal, pelvi-ureteric junction, ureter, vesicoureteric junction or bladder and multiple/bilateral. We did not undertake accurate sample size calculation because of lack of literature concerning the predictors of urological intervention in ED patients presenting with suspected renal colic. Hence, for this exploratory study a sample size of 283 patients was considered reasonable in line with previous studies of renal colic management ${ }^{7}$.

\section{Results}

Two hundred and eighty-two (282) patients were enrolled in the study and 213 (75.5\%) were male and 69 (24.4\%) were female. Mean age of the cohort was 35.02 years (SD 7.9). Sixty percent of the cohort was non-UAE national patients. Impaired renal function was seen in 38 (13.5\%) patients. Renal calculi were confirmed in 108/282 (38\%) patients and the commonest locations of stones were ureter (15\%), ureterovesical junction (12.4\%) and renal (10\%). Seventeen percent of patients had multiple stones and single stone was detected in $24 \%$ of the patients. From the study cohort, 11 (3.9\%) patients were admitted and 6 (2\%) patients underwent urological intervention. Stone size of less than $5 \mathrm{~mm}$ was observed in $30 \%$ of the cohort and stone size of $5-10 \mathrm{~mm}$ was seen in $16 \%$ of the patients. $10 \%$ of patients had stone size of $10 \mathrm{~mm}$ or greater (Table 1). One out of 28 (3.5\%) patients with stone size of greater than $10 \mathrm{~mm}$ underwent urological intervention and one out of 46 (2\%) patients with stone size of 5-10 mm underwent intervention. Four out of six (66.6\%) patients undergoing intervention had stone size of less than $5 \mathrm{~mm}$. Chi square analysis showed no significance association between the stone size and urological intervention. (Table 2) From the six patients receiving intervention (age range: 24-44 years) four had hydronephnosis, five had stones in the ureter and four were UAE national patients. Chi square analysis showed no significant association between presence of hydronephrosis or impaired renal function and urological intervention. (Table 3 \& Table 4) 
Pain commenced suddenly within 6 hours in $55 \%$ of the patients and $80 \%$ of the patients had normal renal function. Dysuria, haematuria and vomiting were present in $20,8.5$ and $7.8 \%$ of the cases respectively. No association was noticed between sudden onset of symptoms, presence of urinary symptoms or vomiting and admission/ urological intervention. Fever was present only in 3 patients and they were not admitted or undergo intervention.

CT showed stones in $38 \%$ of the study cohort and few incidental findings and pathology were noted in $4.6 \%$ of patients such as pancreatitis, diverticulitis and appendicitis, ovarian cyst, cholelithiasis, pyelonephritis. CTKUB was reported normal in rest of the patients

\section{Discussion}

Our study found that of 282 patients who had CTKUB for suspected renal colic, 38\% patients had ureteric calculi presence confirmed. However, urological intervention was performed on six patients; this represented a rate of $2 \%$ for the entire cohort investigated by CTKUB. A previous study ${ }^{5}$ found a higher (8.3\%) rate of urological intervention. Although stone was detected in 108 of 282 patients and 13 patients were found to have other pathologies; some of them incidental in nature. Other patients with normal CT might have had non-radio-opaque stones or non- specific abdominal pain mimicking renal colic as their presenting symptoms

In our study, we found no significant association between the stone sizes, renal function or presenting symptoms and likelihood of urological intervention.

Whilst the usefulness of the CT is undisputable, there is an important trade-off between its impact on treatment plans and the risk of high levels of ionising radiation for the patient. Previous studies have reported increased lifetime attributable risks of cancer to four cancers per 1000 patients for abdomen and pelvic $\mathrm{CT}^{8}$. This is particularly relevant for patients with renal stones as they are susceptible to repeat ED visits and multiple CT scans. ${ }^{9}$ Moreover, CT scans are expensive and prolong patient's length of stay in the busy ED. By curtailing CTKUB use in renal colic patients, there is potential for savings and reducing the length of stay in ED. There is a growing body of opinion, such as the European Association of Urology, that supports ultrasound instead of CT as the preferred initial imaging modality in renal colic patients ${ }^{10}$. A prospective study ${ }^{11}$ recommended that in the setting of negative plain film and ultrasound imaging, CT should be reserved only for the patients not improving on conservative management. In the presence of normal plain film and ultrasound, $90 \%$ of patients had $<5 \mathrm{~mm}$ stones, which passed spontaneously. Furthermore, detection of gynaecological abnormality on CT usually requires further evaluation by other imaging modalities such as ultrasound ${ }^{12}$. A large pragmatic randomised study ${ }^{13}$ of 2759 patients, comparing ultrasound with CTKUB in suspected renal colic reported that the incidence of high risk diagnoses with complications in the first 30 days was low $(0.4 \%)$. Therefore, the authors recommend that CTKUB is not productive as the primary investigation modality for suspected renal colic in ED. In our study, it did not affect the management and urological intervention of the patients 


\section{Limitations}

There were several limitations to this study. The retrospective nature of this study means that all required information may not have been available from the notes review. The limited number of patients enrolled at a single centre may not be fully representative of wider ED populations. Therefore, its results should be interpreted with caution. The data abstractors were not blinded to the outcome of intervention, but this seems unlikely to have led to systematic bias. Although consecutive CTs were selected from the electronic system of ED, patients may have been missed due to either misclassification or may have had only ultrasound prior to intervention. This would be extremely rare in the authors' experience. CT KUB confirmed stone in $38 \%$ of the cohort and other pathologies were noted in $4.6 \%$ of the patients in the study. Thus, CTKUB was reported normal in rest of the cohort. This might be reflection of the local practice of over utilization of CTKUB requests in patients presenting with abdominal pain mimicking renal colic.

\section{Conclusion}

In the vast majority of cases, there was no association between CTKUB findings and the decision to admit or perform urological intervention. Moreover, no significant association could be established between urological interventions and predetermined patients' or radiological variables. Therefore, this study supports the growing consensus that CTKUB is not productive as the primary investigation for suspected renal colic in ED.

\section{Declarations}

\section{Acknowledgement/ Contributors}

$\mathrm{KB}, \mathrm{RA}$, IA conceived the original idea and developed research method and contributed to data collection and abstraction. RC contributed to data collation and interpretation in SPSS. MC and RB supervised and contributed substantially to the development, data interpretation and of final manuscript. All authors contributed in reviewing and providing feedback on the manuscript

\section{References}

1. Chyng-Wen Fwu, Paul W. Eggers et al. Emergency department visits, use of imaging, and drugs for urolithiasis have increased in the United States, Kidney International 2013 83, 479-486

2. Elder JW, Delgado MK et al. Variation in the intensity of care for patients with uncomplicated renal colic presenting to US Emergency departments. j emer med 2016; 51:628-635

3. Bultitude M, Rees J. Management of renal colic. BMJ 2012; 345: e5499

4. Westphalen AC, Hsia RY et al. Radiological imaging of patients with suspected urinary track stones: national trends, diagnoses, and predictors. Acad Emerg. Med. 2011; 18: 699-707 
5. Blecher G, Meek R et al. Predictors for urological intervention and alternate diagnoses in people having computed tomography urography for suspected renal colic. Emergency Medicine Australasia 2016; 28: 56-61

6. Papa L, Steil IG et al. Predicting intervention in renal colic patients after emergency department evaluation. CJEM 2005; 7: 78-86

7. Zwank MD, Ho BM et al. Does computed tomographic scan affect diagnosis and management of patients with suspected renal colic? American Journal of Emergency Medicine 2014; 32: 367-370

8. Smith-Bindman R, Lipson $\mathrm{J}$ et al. Radiation dose associated with attributable risk of cancer. Arch Intern Med 2009; 169 (22):2078-86

9. Broder J, Lohr $\mathrm{J}$ et al. Cumulative CT exposure in Emergency Department patients evaluated for suspected renal colic. J Emerg Med 2007; 33 (2): 161-8

10. Turk C, Knoll T, et al. Guidelines on Urolithiasis European Association of Urology 2013:1-100.

11. Ripolles Agramunt $M$ et al. Suspected ureteric colic: plain film and sonography vs unenhanced helical CT. A prospective study in 66 patients. Eur Radiology 2004;14: 129-36

12. Patatas $\mathrm{K}$, Panditaratne $\mathrm{N}$ et al. Emergency department imaging protocols for suspected acute renal colic: re-evaluating our service. BJR 2012;85 (1016): 1118-1122

13. Smith-Bindman R, Aubin C, Bailitz J et al. Ultrasonography versus Computed Tomography for Suspected Nephrolithiasis. N Engl J Med 2014;371: 1100-10

\section{Tables}

Due to technical limitations, table 1 is only available as a download in the Supplemental Files section.

\section{Stone size and Intervention Table 2}

\begin{tabular}{|llll|}
\hline Chi-Square Tests & & & \\
\hline Vearson Chi-Square & $5.776^{\mathrm{a}}$ & 4 & .217 \\
\hline Likelihood Ratio & 7.647 & 4 & .105 \\
\hline Linear-by-Linear Association & 4.959 & 1 & .026 \\
\hline N of Valid Cases & 282 & & \\
\hline
\end{tabular}

\section{Hydronephrosis and Intervention Table 3}




\begin{tabular}{|llll|}
\hline Chi-Square Tests & & & \\
\hline Vearson Chi-Square & $2.385^{\mathrm{a}}$ & 2 & .303 \\
\hline Likelihood Ratio & 3.084 & 2 & .214 \\
\hline Linear-by-Linear Association & .004 & 1 & .951 \\
\hline N of Valid Cases & 282 & & \\
\hline
\end{tabular}

Impaired renal function and Intervention Table 4

\begin{tabular}{|llll|}
\hline Chi-Square Tests & & & \\
& Value & df & Asymptotic Significance (2-sided) \\
\hline Pearson Chi-Square & $2.355^{\mathrm{a}}$ & 2 & .308 \\
\hline Likelihood Ratio & 2.272 & 2 & .321 \\
\hline Linear-by-Linear Association & .058 & 1 & .810 \\
\hline N of Valid Cases & 282 & & \\
\hline
\end{tabular}

\section{Supplementary Files}

This is a list of supplementary files associated with this preprint. Click to download.

- Table1.jpg 